\title{
Proliferative Verrucous Leukoplakia
}

National Cancer Institute

\section{Source}

National Cancer Institute. Proliferative Verrucous Leukoplakia. NCI Thesaurus. Code C4696.

Warty leukoplakia on the oral mucosa. It is associated with a high risk for malignant transformation. 